\title{
CRESCIMENTO EM ALTURA DE Pinus elliottii ENGELM., NA REGIÃO DE PIRATINI NO RIO GRANDE DO SUL, BRASIL
}

\author{
HEIGHT GROWTH OF Pinus elliottii ENGELM., IN THE REGION OF PIRATINI, \\ RIO GRANDE DO SUL STATE, BRAZIL
}

\author{
Helio Tonini ${ }^{1}$ César Augusto Guimarães Finger ${ }^{2}$ Paulo Renato Schneider ${ }^{3}$ \\ Peter Spathelf ${ }^{4}$
}

RESUMO

Este trabalho teve como objetivos estudar as tendências de crescimento em altura dominante de árvores localizadas dentro de uma mesma unidade de mapeamento de solo e estudar a viabilidade de se utilizar a posição do relevo como indicador de sítio para árvores de Pinus elliottii, localizadas em Piratini, na região denominada Serra do Sudeste, no Estado do Rio Grande do Sul. Foram coletadas 16 árvores dominantes em diferentes posições do relevo, sendo os dados de altura e idade ajustados através da função de RICHARDS (1959), com três coeficientes. A análise das tendências de crescimento feita através da utilização da análise de covariância e análise gráfica, indicaram que a posição do relevo não foi um bom indicador de sítio, pois houve cruzamentos entre as tendências de crescimento para árvores localizadas na mesma posição de relevo. No entanto, a análise das curvas de crescimento, construídas individual mente para os grupos formados por árvores de mesma tendência de crescimento, indicou que estes cruzamentos, por ocorrerem nas menores idades, não justificariam a construção de mais de um sistema de curvas de índice de sítio para este local e unidade de mapeamento de solo.

Palavras-chave: Pinus elliottii, crescimento em altura, tendência de crescimento.

\section{SUMMARY}

The objective of this work was to study the height growht of Pinus elliottii situated in the Piratini region (southeastern mountain range) in the State of Rio Grande do Sul, Brazil. It was collected 16 dominant trees in different relief positions. The height/age data were adjusted using the function of RICHARDS (1959) with three coefficients and the growth trends were studied by covariance and graphycal analysis. The results

\begin{abstract}
showed that relief was not a good site indicator, because there were intersections of height curves of trees which grew at the same relief position. However, the site index curves were established individually for each group, wihch was formed by tree with the same growth trend,the results showed that these intersections only occur at younger ages and thus do not justify the establishment of another system of site index curves in the region.
\end{abstract}

Key words: Pinus elliottii, height growth, growth trend.

\section{INTRODUÇÃO}

Os ecossistemas funcionam como um complexo ciclo de energia, marcado por perdas e ganhos envolvendo vegetação, atmosfera e solo. Nesse contexto, o cientista florestal dedica-se ao segmento floresta, que é apenas uma parte do ecossistema, e tem como desafio integrar todos os fatores de sítio para produzir uma estimativa da qualidade do sítio florestal.

DAVIS (1966) citou a definição de sítio, dada pela Sociedade Americana de Engenheiros Florestais, como sendo uma área considerada segundo os seus fatores ecológicos em relação a sua capacidade de produzir florestas ou outra vegetação sob a combinação de condições biológicas, climáticas e edáficas.

Para CLUTTER et al. (1983), os métodos para predizer o crescimento de florestas podem ser

\footnotetext{
${ }^{1}$ Engenheiro Florestal, MSc., pelo programa de Pós-graduação em Engenharia Florestal, Centro de Ciências Rurais (CCR), Universidade Federal de Santa Maria (UFSM). E-mail: htonini@ bol.com,br.

${ }^{2}$ Engenheiro Florestal, Doutor, Professor Adjunto do Departamento de Ciências Florestais (DCF), CCR/UFSM, 97105-900, Santa Maria, RS. Autor para correspondência.

${ }^{3}$ Engenheiro Florestal, Dr., Professor Titular, DCF/CCR/UFSM.

${ }^{4}$ Engenheiro Florestal, Dr., Professor Visitante no Programa CAPES/DAAD, DCF/CCR/UFSM
}

Recebido para publicação em 06.06.00. Aprovado em 04.10.00 
classificados em diretos e indiretos. Os métodos indiretos avaliam a qualidade do sítio, a partir de atributos do ambiente, levando em consideração características como clima, solo e vegetação. Os métodos diretos utilizam-se basicamente de indicadores na própria vegetação, que refletem as interações de todos estes fatores de sítio.

$\mathrm{Na}$ prática, os fatores de sítio têm sido menos precisos como indicadores do crescimento potencial, do que predições baseadas em fatores de crescimento, devido à complexidade e ao grande número de variáveis que envolvem esse tipo de levantamento. Devido a isso, a grande maioria das avaliações seguem o segundo caminho, ou seja estimam a qualidade do sítio através de parâmetros obtidos na própria vegetação.

Dentre os fatores de sítio, o relevo, por estar intimamente correlacionado com diferenças microclimáticas, profundidade efetiva do solo, risco de erosão, possibilidade de emprego de mecanização e regime de nutrientes do solo, pode constituir-se em um bom indicador de sítio, uma vez que o crescimento pode ser diferente para árvores que crescem em um mesmo tipo de solo, e estão sujeitas a diferentes exposições e inclinações. SPURR \& BARNES (1973) afirmaram que a classificação topográfica de sítios pode ser feita rapidamente por fotografias aéreas e mapas, sem a necessidade de levantamento de fatores edáficos.

Dentre os parâmetros da vegetação, a altura dominante é a variável dendrométrica que melhor se enquadra nos pré-requisitos para um bom indicador de sítio, que são: facilidade de determinação a campo; possuir boa correlação com a produção volumétrica; indicar a qualidade do sítio para todas as idades do povoamento e ser independente da densidade do povoamento (BURGER, 1976).

Segundo CARMEAN (1970), o índice mais utilizado para determinar a produtividade de uma área florestal é o índice de sítio, que é uma expressão da qualidade do sítio baseada na altura das árvores dominantes e codominantes em uma determinada idade. Entre as diversas definições de altura dominante presentes na literatura, pode-se citar a altura média das 100 árvores mais grossas por hectare (Assmann), e a altura correspondente ao dap médio de $20 \%$ das árvores mais grossas do povoamento (Weise).

Para GIRARDIN \& BROQUEN (1995), os povoamentos que devem ser eleitos, a fim de que se possa utilizar a altura dominante como indicadora das condições de sítio, devem atender aos seguintes pré-requisitos: a população amostrada não deve se encontrar no estado juvenil de crescimento; as densidades não devem ser extremas (entre 900 e 2500 árvores); práticas silviculturais que tenham removido árvores dominantes não devem ter sido realizadas e o estado fitossanitário deve ser bom, ou seja, as árvores não devem ter sofrido danos severos.

A classificação de sítios mediante o estudo do crescimento em altura dominante, para povoamentos plantados com Pinus elliottii, nesta região, tem grande importância, pois através deles se obtêm informações básicas que podem auxiliar na seleção de áreas adequadas (mais produtivas); nas estimativas da produção; na programação e execução de trabalhos de manutenção (limpezas) e desbastes e no planejamento e execução de trabalhos de pesquisa

Dessa forma, este trabalho foi realizado com o objetivo de estudar a viabilidade de se utilizar a posição do relevo, como indicador de sítio para a região da Serra do Sudeste (região de Piratini) no Estado do Rio Grande do Sul e estudar as tendências de crescimento em altura dominante entre árvores localizadas dentro de uma mesma unidade de mapeamento de solo.

\section{MATERIAL E MÉTODOS}

\section{Caracterização da floresta e da área de amostragem}

A área selecionada para a realização deste estudo situa-se no município de Piratini, no Estado do Rio Grande do Sul e apresenta coordenadas de $31^{0} 25^{\prime}$ de latitude sul e $53^{0} 05^{\prime}$ de longitude oeste. Os dados utilizados neste estudo foram obtidos em povoamento implantado com Pinus elliottii, com 18 anos de idade, espaçamento inicial de 2 por $2 \mathrm{~m}$, totalizando 2.500 árvores por hectare e com um desbaste realizado no décimo oitavo ano.

O município de Piratini localiza-se na Unidade de Relevo Planalto Sul-Rio-Grandense, que se caracteriza por apresentar uma estrutura geológica formada por rochas pré-cambrianas, apresentando relevos intensamente dissecados e áreas com relevo fracamente dissecados. As áreas que correspondem aos relevos mais elevados, com altitude ao redor dos 400 metros, onde se localizam, entre outros, o município de Piratini, apresentam-se dissecadas em forma de colina. As áreas intensamente dissecadas estão posicionadas altimetricamente entre as cotas $100 \mathrm{e}$ 200 metros e encontram-se balizando toda a unidade e isolando os relevos ondulados, cujos contatos se fazem em muitos trechos através de escarpas e ressaltos topográficos (HERRMANN \& ROSA, 1990).

Segundo BRASIL, (1973) a área em estudo pertence à Unidade de Mapeamento Pinheiro Machado, que ocupa uma área de $6.000 \mathrm{~km}^{2}$, o que representa 2,22\% da área do Estado. Essa unidade de 
mapeamento caracteriza-se por apresentar solos litólicos distróficos, bem drenados, de coloração escura, apresentando textura média, com porcentagens elevadas das frações mais grosseiras (areia grossa e cascalhos), sendo derivado de granito. Geralmente são solos ácidos, com saturação e soma de bases média, sendo bem providos de matéria orgânica. O horizonte A é de espessura variável, em torno de $50 \mathrm{~cm}$, de coloração escura, franco argilo arenoso, com estrutura maciça, quebrando-se em blocos subangulares.

Para NIMER (1990), o clima predominante na região em estudo foi classificado por Köppen como do tipo Cfa (mesotérmico subtropical com verões quentes e sem estação seca). A temperatura média anual é de $16^{\circ} \mathrm{C}$, com temperatura média do mês mais quente de $24^{0} \mathrm{C}$ e do mês mais frio de $13^{\circ} \mathrm{C}$. A precipitação média anual é de $1500 \mathrm{~mm}$.

\section{Processo de amostragem e obtenção dos dados}

As árvores foram coletadas segundo um gradiente de declividade: árvores de platô (solos eluviais), árvores de encosta superior e encosta média (solos coluviais) e árvores de baixada (solos aluviais).

As unidades amostrais temporárias foram instaladas, com o auxílio do relascópio de Bitterlich de banda estreita, onde as árvores são selecionadas com probabilidade proporcional ao seu diâmetro (amostra por contagem angular). O ângulo selecionado para esse levantamento foi o de $2^{0} 17$ ' 32 ', correspondente ao fator de área basal (FAB) igual a quatro, em que cada árvore selecionada em uma unidade amostral (ACA) representa uma área basal de $4 \mathrm{~m}^{2} /$ ha.

As árvores dominantes foram definidas segundo o conceito de Pollanschütz, descrito por FINGER (1992), como a altura média de Lorey, correspondentes a $12 \mathrm{~m}^{2}$ de área basal por hectare, tomados a partir das maiores árvores. Com base nessa definição, a altura dominante foi determinada pela altura da árvore com diâmetro intermediário entre três árvores.

Em cada ACA, a árvore dominante selecionada foi abatida e dela foram retiradas fatias do tronco nas posições $0,1 \mathrm{~m}, 0,5 \mathrm{~m} 1,3 \mathrm{~m}$ e a partir desta a cada $1,5 \mathrm{~m}$, procurando-se evitar nós e as deformações causadas pelos mesmos nos anéis anuais de crescimento. Os pares de dados, altura e idade, foram obtidos por análise de tronco e com a utilização do software ANATRO desenvolvido por SCHNEIDER (1984).

Foram coletadas 13 árvores dominantes, em diferentes posições do relevo, sendo 4 árvores no platô, 3 na encosta superior, 3 na encosta intermediária e 3 na baixada.

\section{Modelo matemático e procedimento utilizado} para a construção das curvas de índice de sítio

O modelo utilizado para ajustar os dados de altura e idade para cada árvore foi o de RICHARDS (1959), com três coeficientes, que é expresso por:

$h_{d o m}=A \times\left(1-e^{(-k \times t)}\right)^{(1 /(1-M))}$

sendo: $\mathrm{h}_{\mathrm{dom}}=$ altura dominante; $\mathrm{t}=$ idade; $\mathrm{A}, \mathrm{K}$ e

$\mathrm{M}=$ coeficientes do modelo.

Após o ajuste desta função para cada árvore, com o uso do pacote estatístico SAS (1993), utilizou-se a análise de covariância para detectar diferenças em inclinação e níveis entre as curvas das árvores. $\mathrm{Na}$ análise de covariância padrão, primeiramente, testa-se a hipótese da não diferença de inclinação. Se não existir evidência de diferença de inclinação, a hipótese de não diferença de níveis é testada.

Para a construção de curvas padronizadas, utilizaram-se os coeficientes médios $\mathrm{K}$ e $\mathrm{M}$ para cada grupo, e estimou-se o coeficiente $\mathrm{A}$, que foi obtido pela transformação do modelo matemático para uma altura desejada na idade índice, conforme apresentado por FINGER (1991):

$$
A=\frac{I S}{\left(1-e^{(-K x t)}\right)^{(1 /(1-M))}}
$$

sendo: $\mathrm{A}$ = assíntota calculada; IS = índice de sítio; $\mathrm{K}, \mathrm{M}=$ coeficientes; $\mathrm{t}=$ idade índice $; \mathrm{e}=$ base do logaritmo natural.

Com o objetivo de selecionar padrões de crescimento em altura, que se espera o povoamento assuma durante a sua vida, foram construídas curvas de índice de sítio monomórficas em relação aos grupos de mesma tendência de crescimento. Para avaliar graficamente o comportamento das árvores quanto a uma eventual mudança de índice ao longo do tempo, bem como a confiabilidade de se efetuar classificações em idades mais jovens, fez-se o teste de confiabilidade e estabilidade das curvas de índice de sítio. Esse teste foi feito plotando-se as alturas reais de todas as árvores, dentro de cada grupo, sobre as curvas de índice de sítio, sendo que o número de curvas utilizadas nesse teste foi determinado em função dos valores reais de altura atingidos pelas árvores amostras aos 18 anos, ou seja, as curvas de 
crescimento de cada grupo foram ajustadas para passar na mesma altura na idade de 18 anos, permitindo melhor comparar diferenças de crescimento.

\section{RESULTADOS E DISCUSSÃO}

\section{Ajuste do modelo matemático}

O ajuste do modelo de Richards para cada uma das 13 árvores dominantes apresentou alto coeficiente de determinação, explicando $99 \%$ da variação total pela regressão e baixo erro padrão de estimativa em porcentagem, com os valores variando entre $2,27 \%$ e $7,65 \%$ indicando que a dispersão entre os valores observados e estimados pela regressão foi pequena. A análise gráfica entre os valores observados e estimados indicou que o modelo não foi tendencioso, o que é de grande importância na confiabilidade das estimativas.

\section{Estudo das tendências de crescimento em altura dominante.}

A técnica de análise de covariância mostrou, conforme a tabela 1, haver diferenças tanto em nível (árvore), como em inclinação (idade* árvore), uma vez que a hipótese de igualdade de inclinação das curvas de crescimento é rejeitada em nível de $5 \%$ de probabilidade de erro.

$\mathrm{Na}$ tentativa de formar grupos de árvores com mesma tendência de crescimento, que poderiam indicar a necessidade de se fazer curvas separadas para determinadas posições de relevo, utilizou-se o teste de Tukey. No entanto, o agrupamento formado não foi consistente pois, além de incluir árvores em mais de um grupo, a análise gráfica indicou cruzamentos das curvas de crescimento entre árvores consideradas no teste de Tukey como não significativas, que deveriam ser paralelas.

A análise gráfica das curvas de crescimento por posição do relevo indicou que as árvores localizadas na mesma posição de relevo não apre-

Tabela 1 - Análise de covariância para as árvores dominantes, Piratini, RS, 200.

\begin{tabular}{lrrcrr}
\hline FV & GL & \multicolumn{1}{c}{ SQ } & QM & F & Pr>F \\
\hline Coeficientes & 3 & 7885,5604 & $2628,5201 *$ & 8606,81 & 0,0001 \\
Árvore & 12 & 321,3398 & $26,7783^{*}$ & 87,69 & 0,0001 \\
Idade*Árvore & 12 & 31,6148 & $2,6345^{*}$ & 8,62 & 0,0001 \\
Erro & 181 & 55,2746 & 0,3054 & & \\
Total & 208 & 8293,7896 & & & \\
\hline
\end{tabular}

FV: Fonte de variação; GL: Graus de liberdade; SQ: Soma de quadrados; QM: Quadrado médio; F: Valor de F calculado, Pr>F: Probabilidade de significância do valor de F.

* Significativo em nível de 5\% de probabilidade de erro. sentaram um padrão semelhante de desenvolvimento em altura, havendo cruzamentos entre as curvas de crescimento para árvores localizadas na mesma posição de topográfica. A causa da variação na forma de crescimento dentro da posição relativa no terreno não pode ser apurada com as observações tomadas a campo por ocasião da coleta dos dados (exposição e posição do relevo). Como hipótese, pode-se considerar como decorrente de variações locais de solo, disponibilidade de água, entre outros. A respeito desse assunto, COILE (1952) afirmou que o grau de extensão da declividade, influi no movimento de água superficial e subterrânea, e que declividades suaves têm um maior potencial de abastecimento de água, do que fortes declividades com a mesma precipitação.

Uma vez que as árvores localizadas em uma mesma posição do relevo não apresentaram padrão semelhante de desenvolvimento em altura, tornou-se necessário fazer grupos com árvores de mesma tendência de crescimento, sendo essas árvores selecionadas de acordo com o paralelismo das curvas de crescimento (Figura 1). Através desse procedimento, foi possível separar as árvores em três grupos distintos: o grupo 1 foi formado pelas árvores $1,4,6,7,8,9,10,12$ e 13; o grupo 2 foi formado pelas árvores 2 e 11, e o grupo 3 foi formado pelas árvores 3 e 5.

O grupo 1 foi formado por árvores que cresciam no platô, encosta superior, encosta média e baixada em diferentes exposições. O grupo 2 foi formado por uma árvore de platô e uma de encosta média, e o grupo 3 foi formado por duas árvores de platô.

Após a definição dos grupos, fez-se com o uso dos coeficientes médios $\mathrm{A}, \mathrm{K}$ e $\mathrm{M}$ uma curva média para cada grupo, com o objetivo de analisar as tendências médias de crescimento entre os grupos (Figura 2). A análise dessa figura permite a comprovação da diferença significativa de inclinação detectada na análise de covariância (Tabela 1), na qual pode se observar cruzamentos entre os grupos $1 \mathrm{e}$ 2 , sendo que o grupo 3, formado por árvores de platô, apresentou maior crescimento em altura em todas as idades analisadas.

Como houve diferença gráfica de inclinação entre os três grupos formados, foram feitas curvas separadas para cada grupo, utilizando-se os coeficientes médios K e M. Para o grupo 1, os valores de K e M utilizados foram 0,06205 e 0,23577; para o grupo 2, estes valores foram de 0,02773 e 0,15379 e, para o grupo 3 , o valores destes coeficientes foram 0,04985 e 0,23065 respectivamente.

O teste de confiabilidade e estabilidade pode ser observado na figura 3 , o qual indicou 


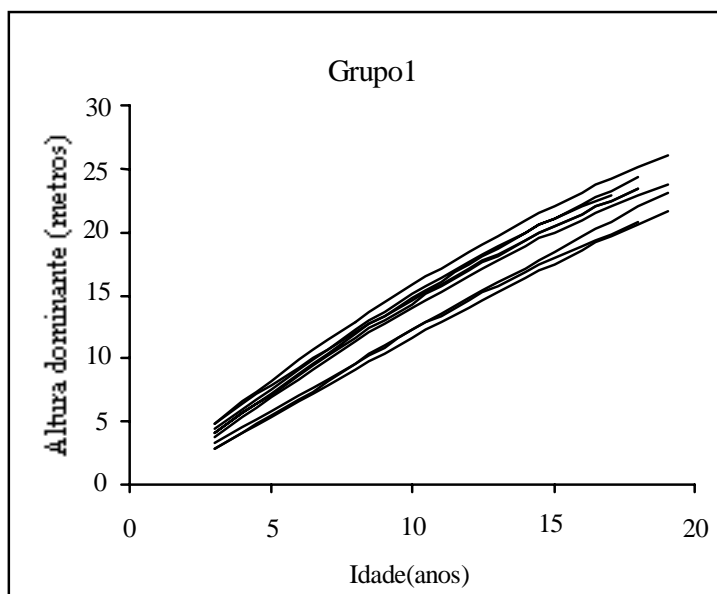

Grupo2

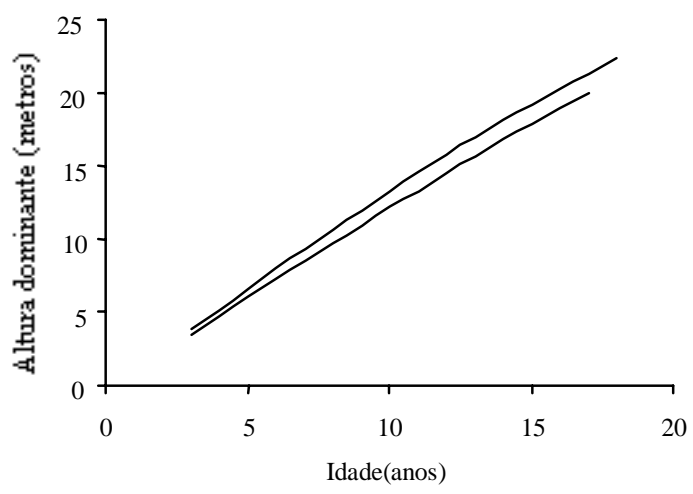

Grupo3

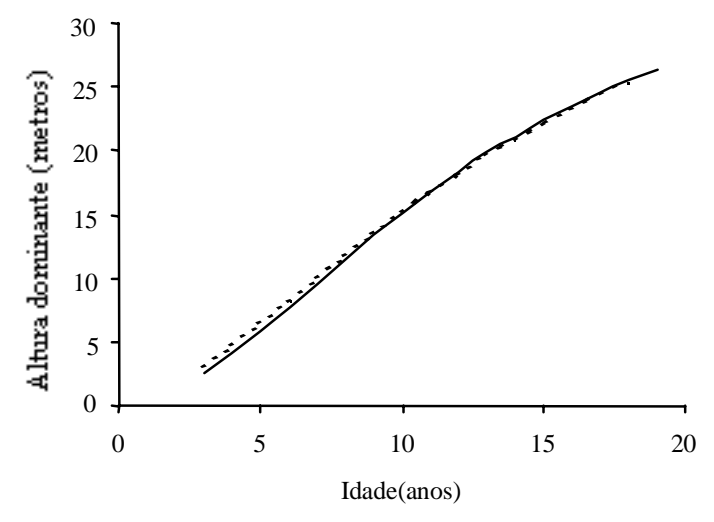

Figura 1 - Grupos formados por árvores com semelhantes tendências de crescimento. Piratini, RS, 2000.

que em todos os grupos houve uma instabilidade das árvores até a idade dos 8 anos, provavelmente ocasionada pela adaptação da planta ao local de crescimento, portanto, deve-se observar que o uso dessas curvas, se os povoamentos forem muito jovens, pode trazer alguma imprecisão na classificação de sítios.

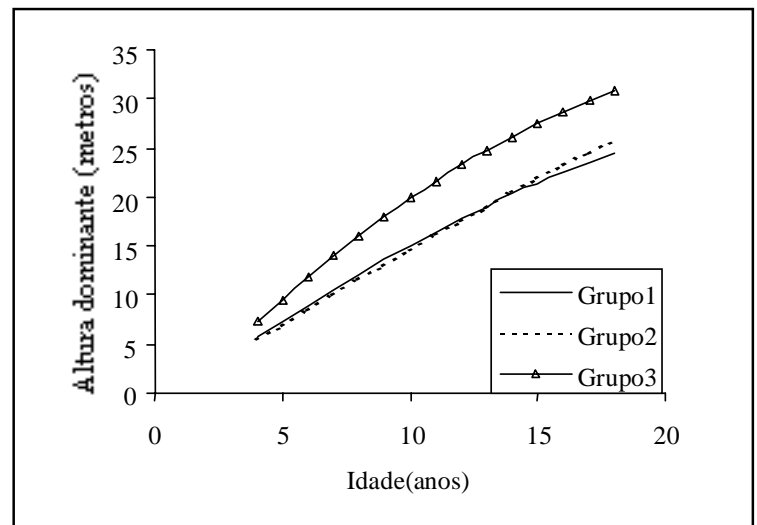

Figura 2 - Tendências médias de crescimento entre os grupos. Piratini, RS, 2000.

Para idades acima dos 8 anos, verifica-se uma boa estabilidade, indicando que as árvores tendem a permanecer com mesma tendência de crescimento, o que é indispensável nos estudos de crescimento e produção. Esse resultado vem de encontro aos resultados obtidos por SCOLFORO \& MACHADO (1988), MARCOLIN (1990) e SELLE (1993). No entanto, para esses autores, a estabilização no padrão de desenvolvimento em altura ocorreu um pouco mais cedo, por volta dos cinco anos, o que provavelmente deve-se às melhores condições de crescimento encontradas naquelas áreas de estudo, nas quais, devido a uma taxa de crescimento inicial mais acelerada, a definição e estabilização no crescimento em altura ocorreu mais cedo.

Como o teste de confiabilidade e estabilidade indicou que os grupos foram formados de forma eficiente, a comparação entre as curvas monomórficas construídas para cada grupo fez-se necessária. Dessa forma, pode-se conhecer a magnitude dos erros de prognose em altura cometidos ao se adotar um sistema único de curvas, uma vez que, por razões práticas, não teria sentido adotar mais de um sistema para um mesmo local, pois não houve uma relação nítida entre o relevo e a tendência de crescimento. Essa comparação foi feita utilizando-se um mesmo índice de sítio por grupo de tendência de crescimento como pode ser observada na figura 4.

A análise da figura 4 indicou que o padrão no crescimento em altura para os três grupos formados foi semelhante. A adoção de um único sistema de curvas de índice de sítio para os grupos 2 e 3 pode ser perfeitamente viável, uma vez que não existem diferenças gráficas significativas de prognose entre esses dois grupos. Entre os grupos 1 e 3, o erro máximo de prognose em altura cometido será de 1.1 metros, tendendo a diminuir com a idade chegando a valores nulos próximo aos 17 anos. 


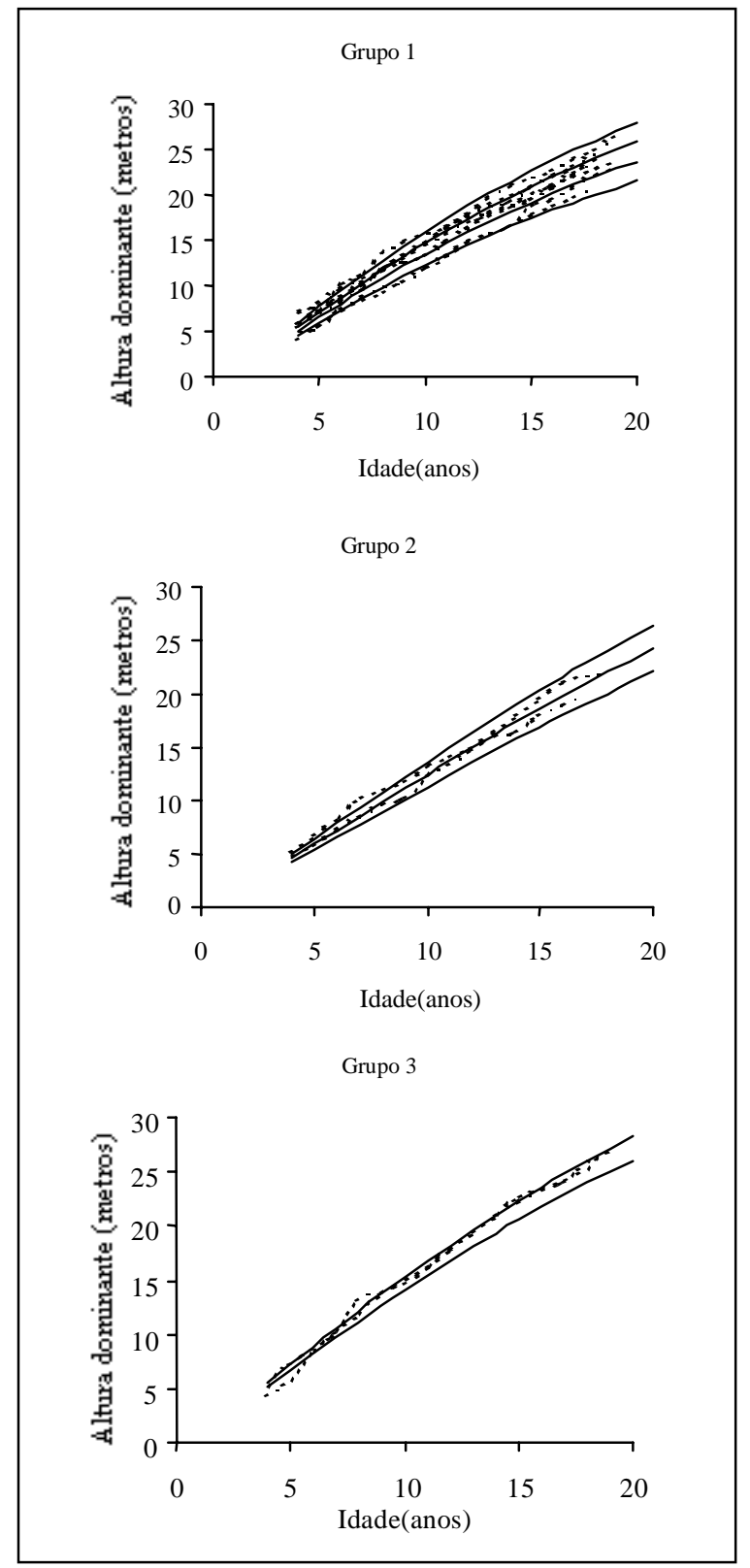

Figura 3 - Confiabilidade e estabilidade das curvas de índice de sítio para grupos de mesma tendência de crescimento. Piratini, RS, 2000.

Uma vez que são esperados erros de até $0,60 \mathrm{~m}$ em medições de altura feitas a campo em condições ideais, o erro cometido ao se adotar um sistema único de curvas para esse local torna-se aceitável, podendo-se optar por um único sistema de curvas do tipo monomórfico para descrever o crescimento em altura nessa localidade.

\section{CONCLUSÕES}

De acordo com os resultados obtidos neste estudo pode-se concluir que:

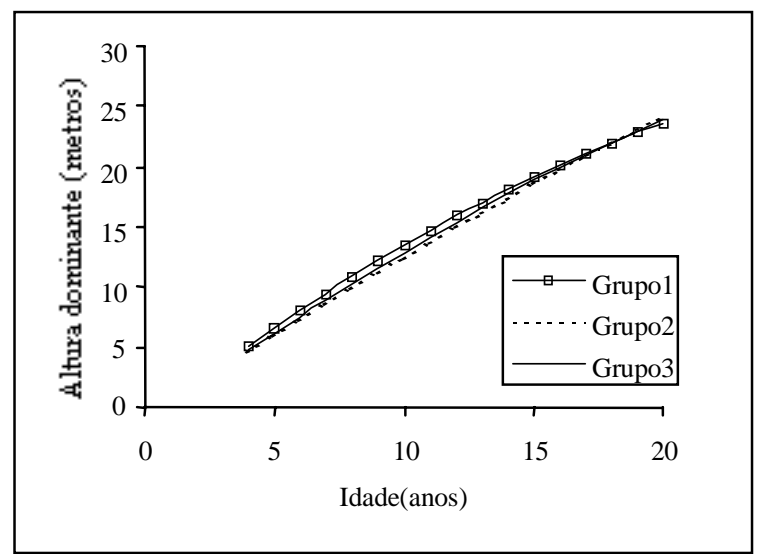

Figura 4 - Comparação entre curvas de índice do sítio construídas para os grupos de árvores com mesma tendência de crescimento. Piratini, RS, 2000.

A função de Richards com três coeficientes descreveu com precisão o crescimento em altura dominante em função da idade para todas as árvores analisadas.

A posição do relevo, de uma forma geral, não foi um bom indicador de sítio, uma vez que houve cruzamentos das curvas de crescimento entre árvores localizadas na mesma posição topográfica.

O agrupamento gráfico visual mostrou ser eficiente na formação de grupos com semelhantes tendências de crescimento.

Apesar da análise de a covariância indicar a existência de diferenças de inclinação entre as curvas de crescimento em altura para árvores individuais, o padrão no crescimento em altura para o povoamento, obtido através das curva de índice de sítio, foi semelhante, indicando que um único grupo de curvas monomórficas pode ser construído para este local.

\section{AGRADECIMENTOS}

Os autores agradecem à empresa Germina Agroflorestal Ltda, localizada no município de Piratini, pela permissão para o levantamento dos dados e abate das árvores em áreas de sua propriedade, sem os quais não seria possível a realização deste trabalho.

\section{REFERÊNCIAS BIBLIOGRÁFICAS}

BRASIL. Levantamento de reconhecimento dos solos do Estado do Rio Grande do Sul. Recife, 1973. 431p.

BURGER, D. Ordenamento florestal I. 2.ed. Curitiba : UFPR, 1976. np.

CARMEAN, W.H. Tree-growth patterns in relation to soil and site. Tree growth and forest soils. USA : Oregon State University, 1970. 527p.

Ciência Rural, v. 31, n. 3, 2001. 
COILE, T.S. Soil and growth of forests. Advances in Agronomy, p.329-359, 1952

CLUTTER, J.L, FORTSON, J.C, PIENAAR, L.V. Timber management: a quantitative approach. USA : John Wiley \& Sons, 1983. 329p.

DAVIS, K.P. Forest managment, regulation and valuation. USA : Mc Graw Hill, 1966. 519p.

FINGER., C.A.G. Ein Beitrag zur ertragskunde von Eucaliptus grandis und Eucalyptus saligna in südbrasilien. Wien, Austria, 1991. 136p. Tese (Doutourado em Manejo Florestal) - Universität für Bodenkultur, 1991.

FINGER, C.A.G . Fundamentos de biometria florestal. Santa Maria : UFSM, 1992. 269p.

GIRARDIN, J.L, BROQUEN, P. El crecimiento de Pinus ponderosa Dougl. Y Pseudotsuga menziessii Mirb. En diferentes condiciones de sítio. Bosque, v.16, n.2, p.57-67. 1995.

HERRMANN, M.L.P, ROSA, R. Relevo. In: IBGE. Fundação Instituto Brasileiro de Geografia e Estatística. Geografia do Brasil: Região Sul. Rio de Janeiro: 1990. p.59-83.

MARCOLIN, M. Polimorfismo e tamanho da amostra para construção de curvas de índice de sítio para Pinus taeda L., no Segundo Planalto Paranaense. Curitiba, 1990. 57p. Dissertação (Mestrado em Engenharia Florestal)
Universidade Federal do Paraná, 1990.

NIMER, E. Clima. In: IBGE. Fundação Instituto Brasileiro de Geografia e Estatística. Geografia do Brasil: Região Sul. Rio de Janeiro, 1990. p151-187.

RICHARDS, F.J. A flexible growth function for empirical use. Journal of experimental botany, v.10, n.29, p.290-300. 1959.

SAS - STATISTICAL ANALYSIS SYSTEM: Programa de computador, ambiente VM. Cary, 1993. Versão 6.08.

SCHNEIDER, P.R. ANATRO: Programa de computador, ambiente MS-DOS. Santa Maria, 1984.

SCOLFORO, J.R.S, MACHADO, S. A. Curvas de índice de sítio para plantações de Pinus elliottii nos Estados do Paraná e Santa Catarina. Revista Floresta, Curitiba, v.17, p.140-158, 1988 .

SELLE, G.L. Influência dos fatores ambientais na classificação de sítio para Pinus taeda $L$, na região de Cambará do Sul, RS, Brasil. Santa Maria, 1993. 85p. Dissertação (Mestrado em Engenharia Florestal) - Programa de Pós-graduação em Engenharia Florestal, Universidade Federal de Santa Maria, 1993.

SPURR, S.H, BARNES, B.V. Forest ecology. New York : Ronald, 1973. 571p.

Ciência Rural, v. 31, n. 3, 2001. 